\title{
The Influence of Frequency of AC Component on Space Charge Behaviours in Polyethylene under Combined AC and DC Electric Fields
}

\author{
C. Zhou and G. Chen \\ The Tony Davies High Voltage Laboratory, University of Southampton \\ Southampton SO17 1BJ, United Kingdom \\ *E-mail: cz3g13@ecs.soton.ac.uk
}

\begin{abstract}
Combined ac and de voltages may occur in high voltage power transmission system, which bring challenges to the dielectric materials. Generally speaking, space charge is well recognised under high voltage dc conditions but has received little attention under high voltage ac conditions. Under ac electric fields, the formation space charge in the material depends strongly on the frequency. The knowledge of space charge behaviour in dielectric materials under combined voltages is desirable in terms of both experimental and simulation. In this paper, a numerical model based on the bipolar charge injection/transport model is used to analyse characteristics of space charge in polyethylene under combined electric fields at room temperature. The DC component ratio and the overall root mean square (RMS) are kept the same, while the frequency of the $\mathrm{AC}$ component is altered from $0.05 \mathrm{~Hz}$ to $50 \mathrm{~Hz}$. It has been found that the amount of the accumulated charges reduces with increasing frequency due to the more frequent polarity reversal. Moreover, the time to reach the steady states of charge amount increases with frequency as well.
\end{abstract}

\section{INTRODUCTION}

Detailed knowledge of electric field or space charge distribution in dielectrics is necessary for the understanding of charge storage and transportation. However, owing to the difficulty and complexity of experimental work, progress has been made over the last 20 years in terms of both charge measurement and numerical methods of analysis of space charge under DC conditions [1-3].

However, the influence of space charge behaviour on insulation degradation under complex electric fields, such as AC and combined AC and DC stresses is still a debated topic, even though the research in this area has been proceeding for a while. There are two possible reasons. Firstly, previous experimental measurements showed small amount of space charges gathered under AC electric fields, and the accumulated amount is further reduced under high frequencies conditions [4, 5]. Therefore, space charge under complex electric stresses is assumed to be less harmful than that under a dc field and attracts much less research attention. Secondly, the reliable detection of space charge under complex stresses is much harder comparing with measurement under DC fields due to constant variation of voltage with time. This paper mainly focuses on the influence of frequency of $\mathrm{AC}$ component within the combined electric fields on the space charge distribution in polyethylene. A numerical model using bipolar transportation mechanism adopted from modelling space charge under purely AC [6] and DC [7] is chosen to compute the charge dynamic under combined condition with same $\mathrm{AC}$ and $\mathrm{DC}$ composition ratio while changing $\mathrm{AC}$ component frequency from $0.05 \mathrm{~Hz}$ to $50 \mathrm{~Hz}$.

\section{NUMERICAL MODELLING}

There are the three basic equations used in the bipolar charge transport simulations in the insulation under combined $\mathrm{AC}$ and DC conditions. They are Poisson's equation (Eq1), Transport equation (Eq2) and Continuity equation (Eq3), where electric field is calculated by the Poisson's equation, current density is computed using Transport equation, and the influences of traps and recombination effects are introduced by the Continuity equations $[3,8]$.

$$
\begin{gathered}
\frac{\partial E(x, t)}{\partial x}=\frac{\rho(x, t)}{\varepsilon} \\
J(x, t)=\mu n(x, t) E(x, t) \\
\frac{\partial n(x, t)}{\partial t}+\frac{\partial J(x, t)}{\partial x}=s
\end{gathered}
$$

Where $\rho$ is the net charge density; $\varepsilon$ is the permittivity of the dielectric; and $s$ is the source term.

The Continuity equation is solved by splitting methods, and Eq3 is split into two, Eq4 and Eq5, where Eq4 is homogeneous while Eq5 is of only one variable and constant coefficients.

$$
\begin{gathered}
\frac{\partial n(x, t)}{\partial t}+\frac{\partial J(x, t)}{\partial x}=0 \\
\frac{\partial n(x, t)}{\partial t}=s
\end{gathered}
$$

The source term is designed to present the effects of charge recombination and trapping phenonina, and in consideration of each species (mobile/trapped electrons/holes) the Eq5 should be presented in four terms.

$$
\begin{gathered}
s_{1}=\frac{\partial n_{e \mu}}{\partial t}=-S_{1} n_{h t} n_{e \mu}-S_{3} n_{h u} n_{e \mu}-B_{e} n_{e \mu}\left(1-\frac{n_{e t}}{n_{0 e t}}\right) \\
s_{2}=\frac{\partial n_{h \mu}}{\partial t}=-S_{2} n_{e t} n_{h \mu}-S_{3} n_{h u} n_{e \mu}-B_{h} n_{h \mu}\left(1-\frac{n_{h t}}{n_{0 h t}}\right) \\
s_{3}=\frac{\partial n_{e t}}{\partial t}=-S_{2} n_{h \mu} n_{e t}-S_{0} n_{h t} n_{e t}+B_{e} n_{e \mu}\left(1-\frac{n_{e t}}{n_{0 e t}}\right) \\
s_{4}=\frac{\partial n_{h t}}{\partial t}=-S_{1} n_{h t} n_{e \mu}-S_{0} n_{h t} n_{e t}+B_{h} n_{h \mu}\left(1-\frac{n_{h t}}{n_{0 h t}}\right)
\end{gathered}
$$


Where $s_{1}, s_{2}, s_{3}, s_{4}$ are the source term for each species; $S_{0}$, $S_{1}, S_{2}, S_{3}$ are the recombination coefficients; $B_{e}$ and $B_{h}$ are the trapping coefficients for electrons/holes; $n_{e \mu}, n_{e t}, n_{h \mu}, n_{h t}$ respectively indicate the densities of each species: mobile electrons, trapped electrons, mobile holes and trapped holes; n0et, n0ht are indicating the trap densities for electrons and holes. The detailed meaning of these parameters is illustrated below in Figl:

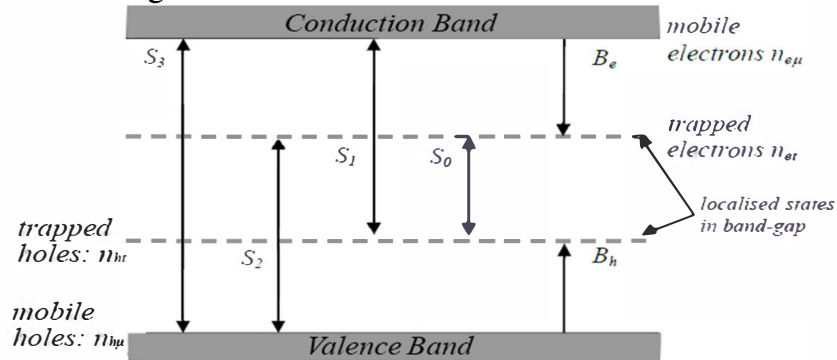

Fig.1 Trapping and recombination of bipolar charge carriers [6]

Besides, the charge carriers' conduction process is characterised using the mobility of the charge carriers within the Transport equation (Eq2). As the electrons and holes may have their own field dependence [9], the mobility of them are computed differently. The mobility for electrons is analysed using Power's law, as deduced in Eq7 [10]. And the mobility of holes is predicted utilising a curve-fitting function of an experimental result of Positive charge velocity charging along with the electric field increasing (Fig.2 (a)) [11]. Details are demonstrated below:

$$
\begin{gathered}
J=\mu n E=v n \\
v=\mu_{0} E^{m_{1}} \text { (Electrons) } \\
v=\left\{\begin{array}{c}
a_{1} E+a_{2} E^{2}+a_{3} E^{3}\left(0<E \leq E_{c}\right) \text { (Holes) } \\
b E^{-m_{2}}\left(E>E_{c}\right)
\end{array}\right.
\end{gathered}
$$

Where $J$ is the conduction current density; $\mu$ is the mobility of charge carrier and is field dependent; $v$ is the drift velocity; $\mu_{0}$ is the mobility under low electric fields; $m_{1}, m_{2}$ are the power indexes; $n$ is the density of mobile species; and $E$ is the local electric field; $\mathrm{a}_{1}, \mathrm{a}_{2}, \mathrm{a} 3, \mathrm{~b}$ and $m_{2}$ are the constants obtained from curve-fitting of Fig. 2.(a) $\left(a_{1}\right.$ is $1.746 \times 10^{-14}, a_{2}$ is $-3.141 \times 10^{-22}$, a 3 is $1.454 \times 10^{-30}, \mathrm{~b}$ is 19.51 and $m_{2}$ is 1.065$)$; Ec is a critical field distinguishing the low and high electric field phenomena for positive charge carriers. In Fig. 2 (a), its value is $100 \mathrm{kV} / \mathrm{mm}$.

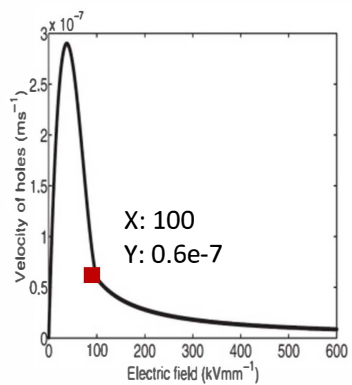

(a)

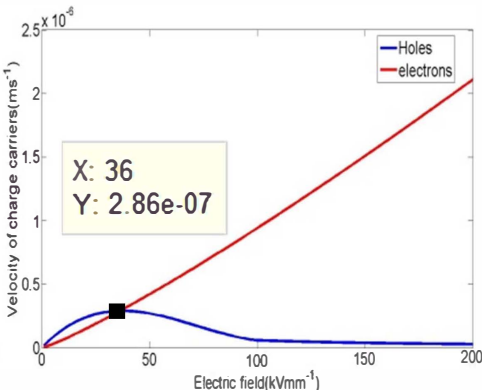

(b)
Fig. 2 Measured holes' velocity versus fields and used velocities versus fields (a)is measured results of Chen [11] and (b) is the used velocities of holes and electrons in the simulation
Furthermore, charges within the insulation are assumed to be sourced by Schottky injection (Eq8) and the extraction of charge from the electrodes can be calculated using Transport equation, presented in Eq9.

$$
\begin{aligned}
& J_{e}(0, t)=A T^{2} \exp \left(\frac{-e w_{e i}}{\mathrm{kT}}\right) \exp \left(\frac{e}{\mathrm{kT}} \sqrt{\frac{e E(0, t)}{4 \pi \varepsilon}}\right) \\
& J_{h}(d, t)=A T^{2} \exp \left(\frac{-e w_{h i}}{\mathrm{kT}}\right) \exp \left(\frac{e}{\mathrm{kT}} \sqrt{\frac{e E(d, t)}{4 \pi \varepsilon}}\right)
\end{aligned}
$$

Where $J_{e}(0, t)$ is the injected current density of the electrons at cathode and $\mathrm{J}_{\mathrm{h}}(\mathrm{d}, \mathrm{t})$ is of the holes at anode; $\mathrm{E}(0, \mathrm{t})$ and $E(d, t)$ are the electric fields at cathode and anode respectively; $\mathrm{A}$ is the Richardson constant; $\mathrm{T}$ is the absolute temperature; $\mathrm{e}$ is the elementary charge; $\mathrm{k}$ is the Boltzmann constant; wei and whi are the injection barrier heights for electrons and holes; and $\varepsilon$ is the permittivity of the dielectric.

$$
\begin{aligned}
& J_{e}(d, t)=\mu_{e} E(d, t) n_{e \mu}(d, t) \\
& J_{h}(0, t)=\mu_{h} E(0, t) n_{h \mu}(0, t)
\end{aligned}
$$

Where $J_{e}(d, t)$ and $J_{h}(0, t)$ are the extracted flux of electrons and holes from the electrodes; $\mu$ is the mobility of charge carriers and $n_{e \mu}$ and $n_{h \mu}$ are the density of mobile electrons and holes.

The total current density $J t(x, t)$ is the sum of the conduction current density (first term in Eq10) and the displacement current density (second term in Eq10).

$$
J_{t}(x, t)=J(x, t)+\varepsilon \frac{\partial E(x, t)}{\partial t}
$$

The applied voltage used in the simulation can be expressed as:

$$
V=V_{P} \sin (2 \pi f t)+V_{\text {offsets }}
$$

Where $V_{p}$ is the peak voltage and $f$ is the ac frequency, in this paper, $V_{p}$ is equal to $9 \sqrt{2} \mathrm{kV}, V_{\text {offsets }}$ is $4.5 \mathrm{kV}$, and $\mathrm{f}$ changes from $0.005 \mathrm{~Hz}$ to $50 \mathrm{~Hz}$.

Specific parameter settings are shown in Table I which are in line with previous published work purely HVDC [12] and HVAC [6]. The injection barrier for holes is set lower than the electrons in order to demonstrate the phenomenon holes are easier to inject into the insulation, comparing with electrons. And the velocity of holes and electrons are based on the curves shown in Fig.2.

TABLE I

Parameters for space charge under combined $\mathrm{AC}$ and DC simulation $[6,12]$

\begin{tabular}{|l|l|l|}
\hline Parameter & Value & Unit \\
\hline Barrier height for injection & 1.18 & $\mathrm{eV}$ \\
\hline $\mathrm{W}_{\mathrm{ei}}$ (electrons) & 1.16 & $\mathrm{eV}$ \\
\hline $\mathrm{w}_{\mathrm{hi}}$ (holes) & $4.5 \times 10-16$ & $\mathrm{~m}^{2} \mathrm{~V}^{-1} \mathrm{~s}^{-1}$ \\
\hline Low field Mobility $\mu_{0}$ & 1.165 & $\mathrm{~m}^{2} \mathrm{~V}^{-1} \mathrm{~s}^{-1}$ \\
\hline Power law's index of mobility $\mathrm{n}$ & \multicolumn{2}{|l|}{} \\
\hline Trap density & 100 & $\mathrm{Cm}^{-3}$ \\
\hline $\mathrm{N}_{\text {oet }}$ (electrons) & 10 & $\mathrm{Cm}^{-3}$ \\
\hline $\mathrm{N}_{\text {oht }}$ (holes) & $7 \times 10-3$ & $\mathrm{~s}^{-1}$ \\
\hline Trapping coefficients & $7 \times 10-5$ & $\mathrm{~s}^{-1}$ \\
\hline $\mathrm{B}_{\mathrm{e}}$ (electrons) & \multicolumn{2}{|l}{} \\
\hline $\mathrm{B}_{\mathrm{h}}$ (holes) & $\mathrm{m}^{3} \mathrm{C}^{-1} \mathrm{~s}^{-1}$ \\
\hline Recombination coefficients & $\mathrm{m}^{3} \mathrm{C}^{-1} \mathrm{~s}^{-1}$ \\
\hline $\mathrm{S}_{0}$ trapped electron-trapped hole & $4 \times 10-3$ & $\mathrm{~m}^{3} \mathrm{C}^{-1} \mathrm{~s}^{-1}$ \\
\hline $\mathrm{S}_{1}$ mobile electron-trapped hole & $4 \times 10-3$ & $\mathrm{~m}^{3} \mathrm{C}^{-1} \mathrm{~s}^{-1}$ \\
\hline $\mathrm{S}_{2}$ trapped electron-mobile hole & $4 \times 10-3$ & \multicolumn{2}{|l}{} \\
\hline $\mathrm{S}_{3}$ mobile electron-mobile hole & 0 &
\end{tabular}




\section{Simulation SET-UP AND Results Discussion}

In 2000, Tanaka et al [13] published a paper about the frequency dependence of charge density under AC stress. It showed that the amount of charge within insulation under $\mathrm{AC}$ stress decreases sharply when the frequency is changing from $10^{-3} \mathrm{~Hz}$ to $10^{-2} \mathrm{~Hz}$ and only slightly decreases in other frequency ranges. To clearly show impacts of the different frequencies of $\mathrm{AC}$ component on space charge dynamics, in this paper, the selected external electric fields are including $0.05 \mathrm{~Hz}, 0.5 \mathrm{~Hz}, 5 \mathrm{~Hz}$ and $50 \mathrm{~Hz}$ (power frequency), $4.5 \mathrm{kV}$ (RMS) AC with $4.5 \mathrm{kV} \mathrm{DC}$ voltages

The space charge distribution profiles are simulated by the bipolar model, however, the differences among the curves under various frequencies are difficult to distinguish, due to the limited impact caused by the variable frequencies. Therefore in this section, the distribution curves over spatial coordinate are integrated to obtain the results of total charge amount within the sample altering with time. The integration function used in shown in Eq12.

$$
T C(t)=\int_{0}^{d} \rho(x, t) d x
$$

Where $\mathrm{TC}$ is the total charge amount function changing with time, $d$ is the sample thickness and $\rho$ is the charge density function of time and spatial coordinate.

Fig.3 presents the simulated charge distribution curves under $50 \mathrm{~Hz} 4.5 \mathrm{kV}$ (RMS) AC with $4.5 \mathrm{kV} \mathrm{DC}$ voltages. And Fig.4 presents the distribution curves obtain under $9 \mathrm{kV}$ DC. The time step used for simulation is $0.002 \mathrm{~s}$, and all the charge profiles under $\mathrm{AC}$ and combined $\mathrm{AC}$ and $\mathrm{DC}$ stresses are plotting the phase angle zero of the applied fields.

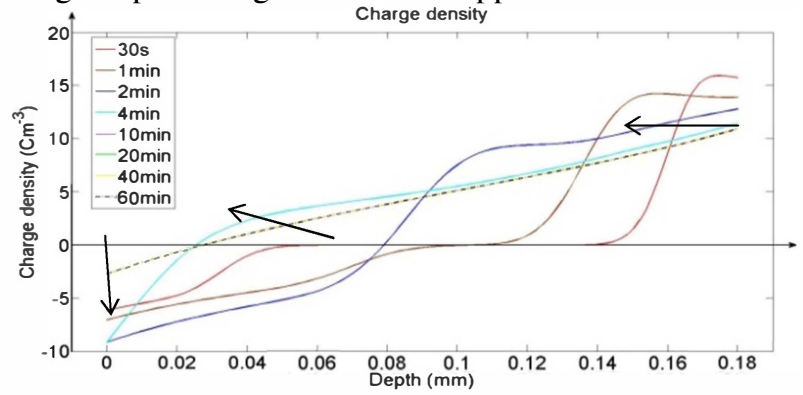

Fig. 3 Charge distribution under $4.5 \mathrm{kV}$ (RMS) $50 \mathrm{~Hz} \mathrm{AC}+4.5 \mathrm{kV}$ DC stress

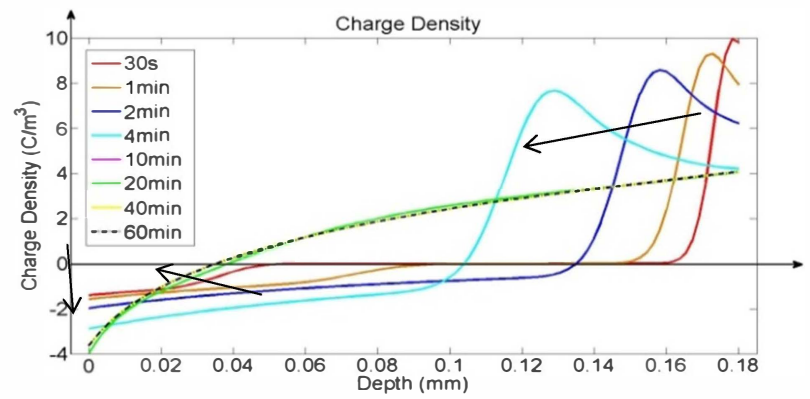

Fig.4 Charge distribution under 9kV DC stress

It can be clearly observed from Fig. 3 that the accumulated positive charges move like a packet towards the opposite electrode with time. Similar to the positive charge movements under the DC condition (Fig.4), due to the influence of $50 \%$
DC component's within the applied field. However the peak values of both accumulated holes and electrons under the combined condition are larger than those under DC condition. This is because the maximum magnitudes of the applied combined fields are larger than that of DC field owing to the AC component, causing a larger amount of charge injections (Schottky injection's exponentially relationship between injected amount and electric fields).

Fig.5 to Fig.7 are the integrated charge of net charge, positive charge and negative charge. Because the diffrereces among the results under high frequencies are too small to distinguish, the high frequency part is zoomed in in a small graph nearby.

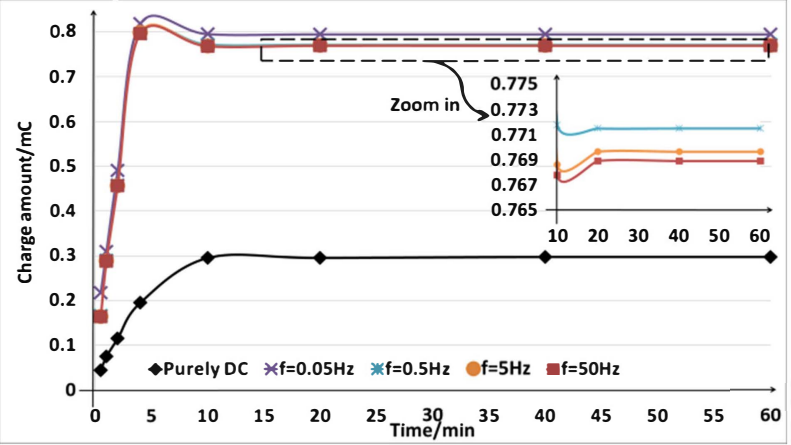

Fig.5 Integral net charge's trend over time under various frequencies

Fig. 5 shows the simulated integrated net charge amount within the insulation under purely DC stress, under combined $\mathrm{AC}$ and $\mathrm{DC}$ stresses with frequency ranging from 0.05 to $50 \mathrm{~Hz}$. It can be clearly observed from the graph that the amounts of net charge under the combined AC and DC stresses are significantly larger than that under the DC conditions. And the net charge amount decreases with frequency increasing, and this phenomenon becomes insignificant when the applied fields' frequency is higher than $0.5 \mathrm{~Hz}$. This inhibition of charge accumulation of applied field's frequency under combined AC and DC conditions is consistent with the experimental results under AC conditions, which is the charge within insulation decreases sharply when the applied fields' frequency is changing from $10^{-3}$ to $10^{-2}$ [4] [13].

Besides, it can also be observed that the amount of net charges within the insulation under combined stress is positive. This is because in the model used it is assumed that holes are easier to inject into the insulation (lower injection barrier) comparing with electrons. Therefore, holes are dominant in the charge accumulation dynamics.

Furthermore, as shown in Fig.5, the charge amount varies dramatically within the first 20 minutes. And a small increase in the transit time can be observed, along with frequency increasing. But the transit time is lengthen under the combined stresses in comparison with that under the pure DC condition. And this is because positive charges are moving slower under the applied combined $\mathrm{AC}$ and $\mathrm{DC}$ conditions, comparing with under the pure DC condition, due to the holes' mobility fielddependent effects (Fig.2). 


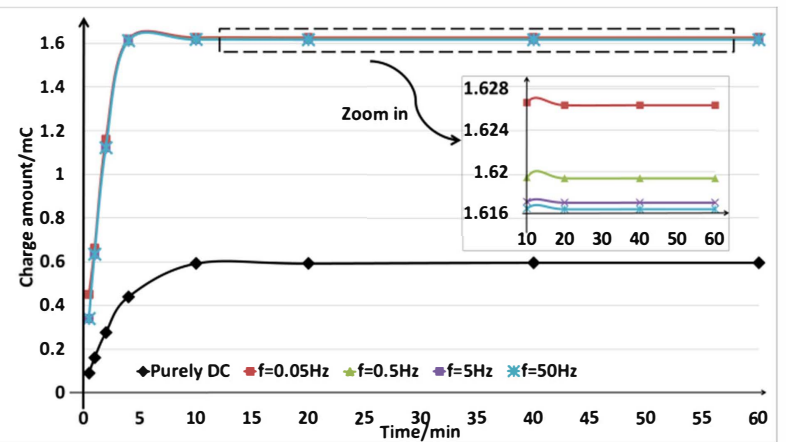

Fig.6 Integral total positive charge's trend over time under various frequencies

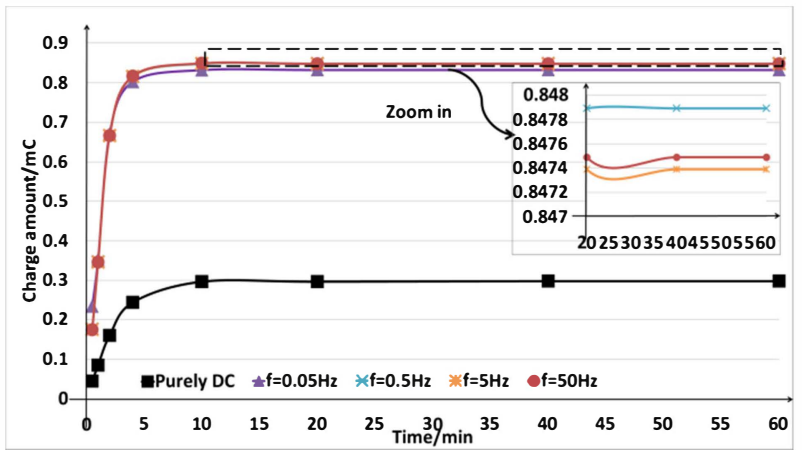

Fig.7 Integral total negative charge's trend over time under various frequencies

Fig.6 and Fig.7 are the integrated results of positive charge and negative charge amounts. Similar to the net charge amount curves, the total positive charges saturates at around 20 minutes, and the higher the frequency, the longer the time to reach the steady state. As comparison, the transient time for electrons under higher frequency $(\geqslant 5 \mathrm{~Hz})$ is generally longer, around 30 to 40 minutes. This indicates the frequency of the applied combined stress has stronger influence on the negative charge movements due to the larger variable range of the mobility of the negative charges than positive charges.

The total positive charge amount and total negative charge amount under combined conditions are also larger than those under purely DC conditions. The overall accumulated positive charge amount is larger than negative amount.

Furthermore, with the frequency increases, the saturated amount of holes decreases. In contrast, this is not the case for electrons. The minimum amount of the saturated electrons occurs at the frequency of $0.05 \mathrm{~Hz}$ instead of $50 \mathrm{~Hz}$. These two graphs also demonstrate the increase of the frequency can weaken the charge accumulation within the insulation under the combined conditions.

\section{CONCLUSION}

In this paper, a bipolar charge transportation model adopting from numerical analysis under HVDC is used to simulate space charge under various frequencies of combined $\mathrm{AC}$ and $\mathrm{DC}$ conditions in order to show the frequency effects of AC Component. The simulated results of space charge distribution and integral net charge, total positive charge and total negative charge are presented.
The results show that the amount of the accumulated charges reduces with the increasing frequency, and the reduction becomes insignificant when the frequency is higher than $0.5 \mathrm{~Hz}$. It is consistent qualitatively with experimental results found under $\mathrm{AC}$ conditions. Besides the frequency of the AC component within the applied electric fields can increase the time to reach the steady state. This effect is also relatively weak when the frequency is high.

This simulation model is an initial one without certification of the experiment. And further modifications are required to be made to the adopted model to coincide with the practical conditions.

\section{REFERENCES}

[1] Y. Li and T. Takada, "Progress in-Space Charge Measurement of Solid Insulating Materials in Japan," Ieee Electrical Insulation Magazine, vol. 10, pp. 16-28, Sep-Oct 1994.

[2] R. J. Fleming, "Space charge in polymers, particularly polyethylene," Brazilian Journal of Physics, vol. 29, pp. 280-294, Jun 1999.

[3] S. Le Roy, G. Teyssedre, C. Laurent, and P. Segur, "Numerical modeling of space charge and electroluminescence in polyethylene under dc field," in Electrical Insulation and Dielectric Phenomena, 2002 Annual Report Conference on, 2002, pp. 172-175.

[4] A. See, J. C. Fothergill, L. A. Dissado, and J. M. Alison, "Measurement of space-charge distributions in solid insulators under rapidly varying voltage using the high-voltage, high-speed pulsed electro-acoustic (PEA) apparatus," Measurement Science \& Technology, vol. 12, pp. 1227-1234, Aug 2001.

[5] G. Chen, M. Fu, X. Z. Liu, and L. S. Zhong, "ac aging and spacecharge characteristics in low-density polyethylene polymeric insulation," Journal of Applied Physics, vol. 97, Apr 152005

[6] J. Zhao, Z. Xu, G. Chen, and P. L. Lewin, "Numeric description of space charge in polyethylene under ac electric fields," Journal of Applied Physics, vol. 108, Dec 152010.

[7] S. L. Roy, P. Segur, G. Teyssedre, and C. Laurent, "Description of bipolar charge transport in polyethylene using a fluid model with a constant mobility: model prediction," Journal of Physics D: Applied Physics, vol. 37, pp. 298-305, 2004.

[8] S. Le Roy, G. Teyssedre, P. Segur, and C. Laurent, "Modelling of space charge, electroluminescence and current in low density polyethylene under DC and AC field," in Electrical Insulation and Dielectric Phenomena, 2004. CEIDP '04. 2004 Annual Report Conference on, 2004, pp. 29-32.

[9] G. Chen and J. W. Zhao, "Observation of negative differential mobility and charge packet in polyethylene," Journal of Physics DApplied Physics, vol. 44, Jun 12011.

[10] J. Zhao, Z. Xu, G. Chen, and P. Lewin, "Effect of field-dependent mobility on current density and dynamics of space charge in polyethylene," in Electrical Insulation and Dielectric Phenomena, 2009. CEIDP '09. IEEE Conference on, 2009, pp. 120-123.

[11] G. Chen, J. Zhao, S. Li, and L. Zhong, "Origin of thickness dependent dc electrical breakdown in dielectrics," Applied Physics Letters, vol. 100, p. 222904, 2012.

J. Zhao, G. Chen, and P. L. Lewin, "Investigation into the formation of charge packets in polyethylene: Experiment and simulation," Journal of Applied Physics, vol. 112, pp. 034116034116-6, 2012.

[13] T. Tanaka, T. Ito, Y. Tanaka, and T. Takada, "Frequency dependence of interfacial space charge formed in laminated dielectrics under AC voltage application conditions," in Electrical Insulation and Dielectric Phenomena, 2000 Annual Report Conference on, 2000, pp. 796-799 vol.2. 